\title{
Any Port in a Storm: Cryptocurrency Safe-Havens during the COVID-19 Pandemic
}

\author{
Shaen Corbet ${ }^{a, b *}$, Yang (Greg) $\mathrm{Hou}^{b}$, Yang $\mathrm{Hu}^{b}$, Charles Larkin ${ }^{c, d, e}$ Les Oxley ${ }^{b}$ \\ ${ }^{a}$ DCU Business School, Dublin City University, Dublin 9, Ireland \\ ${ }^{b}$ School of Accounting, Finance and Economics, University of Waikato, New Zealand \\ ${ }^{c}$ Institute for Policy Research, University of Bath, UK \\ ${ }^{d}$ Trinity Business School, Trinity College Dublin, Dublin 2, Ireland \\ ${ }^{e}$ Kreiger School of Arts Sciences, Johns Hopkins University, Baltimore, MD, USA \\ *Corresponding Author: shaen.corbet@dcu.ie
}

\begin{abstract}
Controlling for the polarity and subjectivity of social media data based on the development of the COVID-19 outbreak, we analyse the relationships between the largest cryptocurrencies and such time-varying realisation as to the scale of the economic shock centralised within the rapidlyescalating pandemic. We find evidence of significant growth in both returns and volumes traded, indicating that large cryptocurrencies acted as a store of value during this period of exceptional financial market stress. Further, cryptocurrency returns are found to be significantly influenced by negative sentiment relating to COVID-19. While not only providing diversification benefits for investors, results suggest that these digital assets acted as a safe-haven similar to that of precious metals during historic crises.
\end{abstract}

Keywords: COVID-19; Pandemic; Sentiment; Cryptocurrency; Safe-haven; Financial Crisis.

\section{Introduction}

The onset of the COVID-19 pandemic has resulted in many variants of international response across social, epidemiological and economic frontiers. The same variation was similarly observed in the response of both financial markets and investors when attempting to evaluate the scale of the issue to which they were confronted. Chinese financial markets acted as the initial epicentre of the shock (Corbet et al. [2020]), however, international contagion effects quickly escalated. In the midst of this confusion, traditional flight-to-safety channels within energy markets dissipated with 
the escalation of geopolitical tensions largely instigated by the US, Russia and Saudi Arabia, with investors struggling to identify credible safe-havens (Goodell [2020], Corbet et al. [2020]). Within this environment, we test the hypotheses as to whether cryptocurrencies acted in this capacity.

One mechanism through which we can proceed is through the use of sentiment data obtained through social media, based on a lexicon of terms developed to quantify the polarity and subjectivity of time-stamped data. Such techniques allow for analysis not only of sentiment based on the severity of the COVID-19 pandemic, but also as to interlinkages between such sentiment and cryptocurrencies as a safe-haven asset in such time of extreme financial market panic. Such research builds on the growing literature that has identified a sharp elevation of cross-asset correlations during the early stages of the pandemic, prior to WHO identification (Corbet et al. [2020]). Cryptocurrencies as a hedging mechanism during periods of financial market chaos, while further validating the product's place as a credible financial market asset, would cause particular concern for regulators and policy-makers due to the wide-ranging issues present with regards to illicit usage (Foley et al. [2019]), manipulation and instability (Gandal et al. [2018]). Despite these concerns, China has continued to educate and support the use of cryptocurrencies through official channels, providing government-produced literature on cryptocurrencies, which has been used to educate the population of China about the nature and future of digital currencies (Corbet et al. [2020]). Such government reassurance could act as both a catalyst and supporting structure to perceived investment safety during market panic.

\section{Data}

We collect data for cryptocurrencies from the Binance exchange, which is the most liquid international exchange throughout 2019 and 2020. Data on the other included assets are collected from Bloomberg. We focus on larger cryptocurrency assets, those with a market value over $\$ 500$ million $^{1}$ as of end January 2020. In Figure 1 and 2, we observe the volatility these assets' prices and volumes traded for the period 1 January 2019 through 31 March 2020 respectively, as separated by the average return and volumes of Bitcoin, altcoins, exchange tokens and privacy coins, with extensive summary statistics between the periods analysed provided in Table 1. As per Corbet et al. [2018], we define returns as the daily log changes and volatility as the five-day standard deviation. In particular, during the onset of the COVID-19 pandemic in the aftermath of the official

\footnotetext{
${ }^{1} \mathrm{~A}$ variety of dates and a number of different market capitalisation values were considered, however, results remained broadly similar. For brevity of presentation, results of these additional analyses are available from the authors upon request.
} 
WHO pandemic declaration on 5 January 2020 and the first case of international transmission on 13 January 2020, there is a pronounced and sustained increase in cryptocurrency volumes traded across each category.

\section{Insert Table $1 \&$ Figures 1 and 2 about here}

The next stage of data collection surrounded the identification of investor sentiment. To complete this task, Twitter data was collected for a period between 1 January 2019 and 31 March 2020 for each of the identified companies. All tweets mentioning the terms 'Covid-19' or 'Covid 19' or 'Coronavirus' or 'Corona Virus', and at the same time the terms 'Cryptocurrency' or 'Crypto' or 'Bitcoin' or 'Blockchain' with inclusive search using the terms 'Financial' or 'Markets' were computationally collected through the search Twitter function on https://twitter.com/explore using the Python 'twitterscraper' package, observing platform rate limiting policies. A total number of 397,558 unique tweets were collected ${ }^{2}$. The data was then aggregated by company and by day as presented in Figure 3, taking sums of the quantitative variables and aggregating the text.

\section{Insert Figure 3 and 4 about here}

We next divide tweets relating to COVID-19 and cryptocurrency based on sentiment. The sentiment variables are based on the Harvard General Inquirer IV-4 dictionary and the Loughran and McDonald Financial Sentiment dictionary. This research focuses specifically on the subjectivity and polarity of the social media analysis, indicative of the scale of real-time understanding of the market as to the severity of the COVID-19 pandemic. Subjectivity analysis of the text is a part of sentiment analysis, where using Natural Language Processing (NLP) researchers classify a text as opinionated or not opinionated. The presence of certain terms such as adjectives, adverbs and some group of verbs and nouns are taken as indicators of a subjective opinion. Thus subjectivity analysis is the classification of sentences as subjective opinions or objective facts. Once through subjectivity analysis we determine whether a sentence is opinionated or not, we perform polarity analysis of the text to ascertain whether it expresses a positive or negative opinion. The purpose of polarity analysis, is to determine the emotional attitude of text writer with respect to the topic under discussion. Each type of sentiment analysed is presented in Figure 4. Each includes the

\footnotetext{
${ }^{2}$ For brevity, additional summary statistics based on these tweets are available from the authors upon request.
} 
following measures to determine sentiment: 1) counts of positive terms; 2) counts of negative terms; 3) a measure of polarity calculated as the number of positive terms minus the number of negative terms divided by the sum of positive and negative terms; and 4) a measure of subjectivity (affect) calculated as the proportion of negative and positive terms relative to the total number of terms in the tweet.

\section{Empirical Approach and Results}

To specifically analyse the effects of COVID-19 sentiment on cryptocurrency returns, we employ a standard GARCH $(1,1)$ methodology of Bollerslev [1986] of the following form:

$$
\begin{gathered}
R_{t}=a_{0}+\sum_{j=1}^{2} b_{j} R_{t-j}+b_{2} V_{t}+b_{3} U S D_{t}+b_{4} \lambda_{t}+\varepsilon_{t} \\
\varepsilon_{t} \mid \Omega_{t} \sim \text { i.i.d. } \quad N\left(0, h_{t}\right) \\
h_{t}=\omega+\alpha_{1} h_{t-1}+\beta_{1} u_{t-1}^{2}
\end{gathered}
$$

$R_{t-j}$ represents the lagged value of the selected cryptocurrency returns, $j$ number of periods before $R_{t}$ is observed. $b_{2} V_{t}$ represents the natural logarithm of cryptocurrency volumes traded, while $b_{3} U S D_{t}$ represents the value of the GBP/USD on the same day on which the cryptocurrency returns were generated. Finally $b_{4} \lambda_{t}$ measures the influence of both the polarity and subjectivity of COVID-19 sentiment respectively. We repeat the analysis for both the Harvard General Inquirer IV-4 and Loughran and McDonald Financial Sentiment dictionaries. Bollerslev [1986] argued for restrictions on the parameters for positivity, $\omega>0, \alpha \geq 0$ and $\beta \geq 0$, and the wide-sense stationarity condition, $\alpha+\beta<1$. While the GARCH $(1,1)$ process is uniquely stationary if $E\left[\log \left(\beta+\alpha \epsilon_{t}^{2}\right)\right]<0$, Bollerslev [1986] also proved that if the fourth order moment exists, then the model can handle leptokurtosis. Bonferroni adjusted results are presented in this analysis. To cater the multiple hypothesis problem, we adjust the significance level using the Bonferroni correction, which leads to a significance level of $0.1 \%$. The generalised Bonferroni method adjusts the significance level such that hypothesis $H_{0,(i)}, i=1, \ldots, s$, is deemed rejected if and only if:

$$
\hat{p}_{(i)} \leq \alpha_{(i)} \equiv k \cdot \alpha / s
$$


This procedure has the advantage of being robust to the dependence structure of the hypothesis tests. The selection of this methodological structure enables robust analysis with regards the influence of negative sentiment relating to the outbreak of COVID-19 on cryptocurrency prices, where significant positive interactions would reflect direct pricing effects as controlled for volumes traded as reflected, and international effects as controlled for through the addition of the GBP/USD exchange rate. The results of the $\operatorname{GARCH}(1,1)$ analysis are presented in Tables 2 and 3 for both polarity and subjectivity respectively. Such structural controls are important due to the wide variety of substantial cryptocurrency events that occurred throughout 2019, such as the Bitcoin flash-crash in late-October. However, during the COVID-19 period analysed, no similar cryptocurrency-related event occurred.

\section{Insert Tables 2 and 3 about here}

While the influence of polarity within this methodological structure is significant and positive throughout the majority of cryptocurrencies analysed, there are particularly pronounced results for altcoins such as Bitcoin SV $(+0.25)$, XRP $(+0.10)$, Stellar $(+0.09)$ and Ethereum $(+0.06)$. Further, exchange tokens and privacy coins both exhibit both pronounced and significant pricing effects in line with the elevating severity of COVID-19. A new influx of liquidity along with increased investor attention could provide one potential explanation for this effect. Testing the effects of the negativity of the response presents far more pronounced influence when focusing on subjectivity. Bitcoin is found to be significantly effected $(+0.30)$ when considering LM subjectivity, while both altcoins and exchange tokens are found to present substantially and significantly elevated estimates when compared to privacy coins. The stability of outcomes across both HI and LM structures presents evidence of methodological robustness.

\section{Conclusions}

When considering the role of negative sentiment relating to the outbreak and development of the COVID-19 pandemic, our results suggest that there developed significant and pronounced timevarying price-volatility effects as investors identified both the severity and nature of the pandemic's growth trajectory and potential economic repercussions. Such results are supported by the continued Chinese-guidance and support of cryptocurrencies as a viable and stable asset class. Such government reassurance could act as both a catalyst and supporting structure to perceived investment safety during a market panic. The use of cryptocurrencies as a safe-haven during episodes 
of severe market volatility will cause alarm within regulatory and policy-making authorities. The widespread lack of continuity, regulation and international legislation, along with the substantially pronounced risks associated with relatively unsophisticated fraud pose serious challenges for policymakers to resolve.

\section{References}

Bollerslev, T. (1986). Generalized autoregressive conditional heteroskedasticity. Journal of Econometrics 31(3), 307-327.

Corbet, S., Y. Hou, Y. Hu, B. Lucey, and L. Oxley (2020). Aye Corona! The contagion effects of being named Corona during the COVID-19 pandemic. Finance Research Letters, 101591.

Corbet, S., Y. Hou, Y. Hu, and L. Oxley (2020). The influence of the COVID-19 pandemic on asset-price discovery: Testing the case of Chinese informational asymmetry. Available at SSRN.

Corbet, S., Y. Hou, Y. Hu, L. Oxley, and D. Xu (2020). Pandemic-related financial market volatility spillovers: Evidence from the Chinese COVID-19 epicentre. International Review of Economics and Finance (forthcoming).

Corbet, S., C. Larkin, and B. Lucey (2020). The contagion effects of the COVID-19 pandemic: Evidence from Gold and Cryptocurrencies. Finance Research Letters, 101554.

Corbet, S., A. Meegan, C. Larkin, B. Lucey, and L. Yarovaya (2018). Exploring the dynamic relationships between cryptocurrencies and other financial assets. Economics Letters 165, 28-34.

Foley, S., J. R. Karlsen, and T. J. Putniṇš (2019). Sex, drugs, and bitcoin: How much illegal activity is financed through cryptocurrencies? The Review of Financial Studies 32(5), 1798-1853.

Gandal, N., J. Hamrick, T. Moore, and T. Oberman (2018). Price manipulation in the bitcoin ecosystem. Journal of Monetary Economics 95, 86-96.

Goodell, J. W. (2020). COVID-19 and finance: Agendas for future research. Finance Research Letters, 101512. 
Figure 1: Box and Whiskers Plots, Cryptocurrency Returns by Type

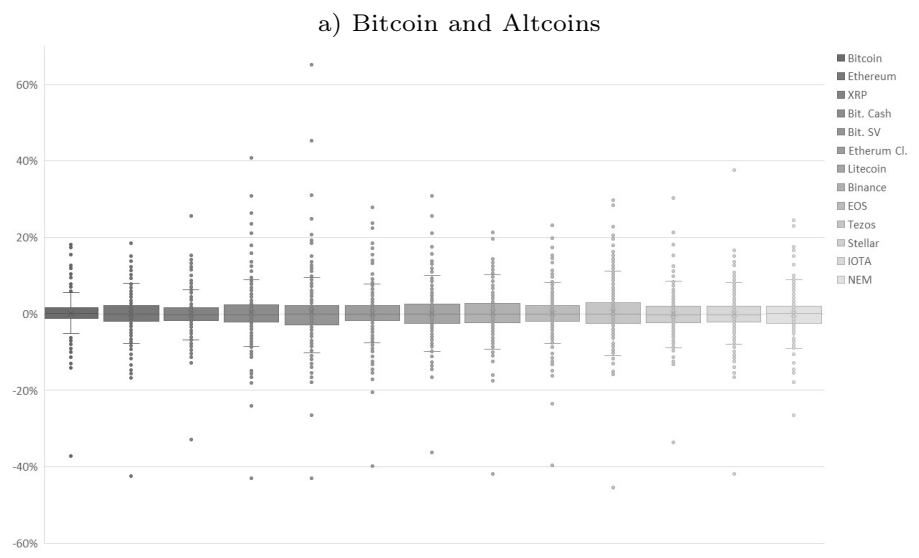

b) Exchange Tokens/Stable Coins
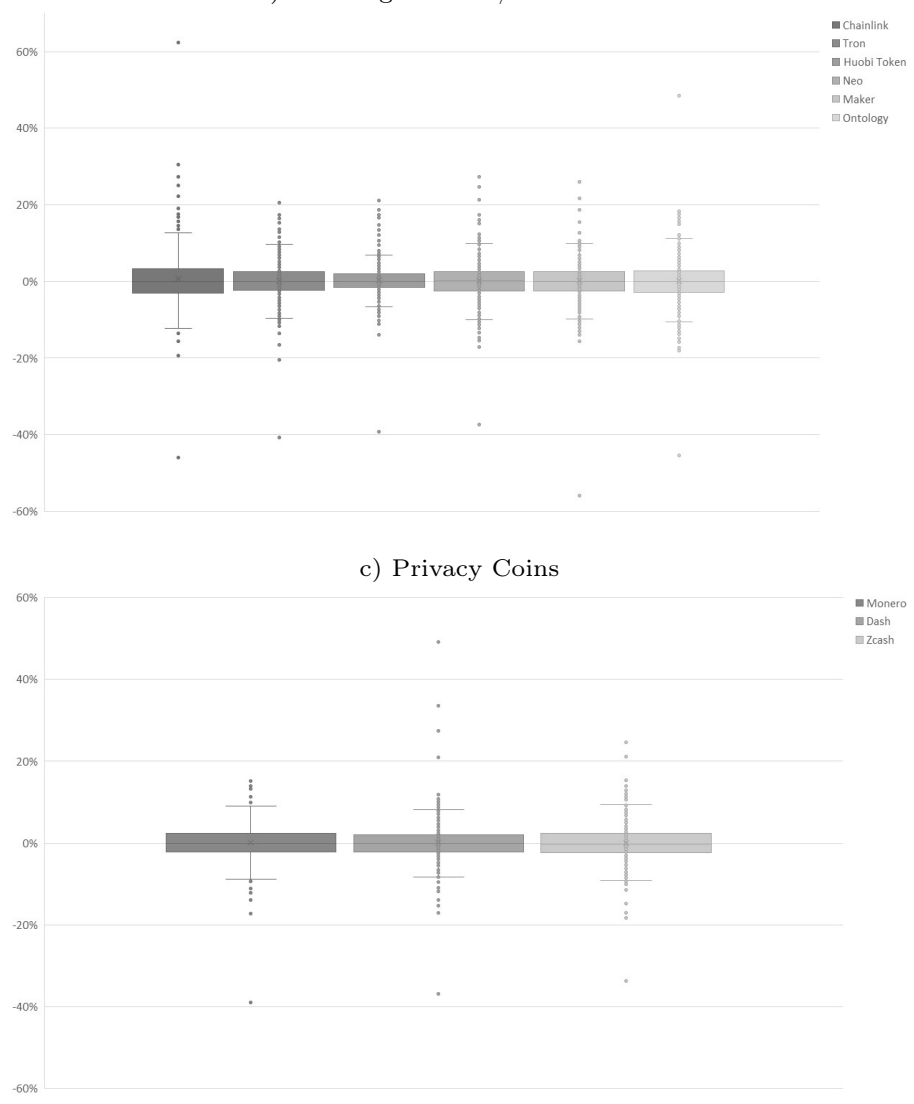

Note: Data is presented for the period 1 January 2019 through 31 March 2020 
Figure 2: Cryptocurrency Volumes Traded by Type

a) Bitcoin and Altcoins

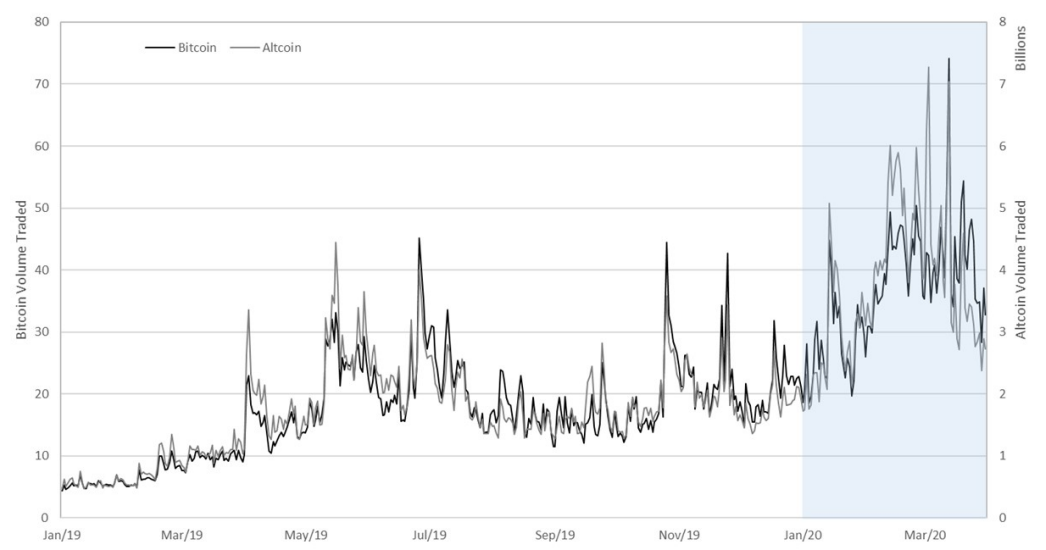

b) Exchange Tokens and Privacy Coins

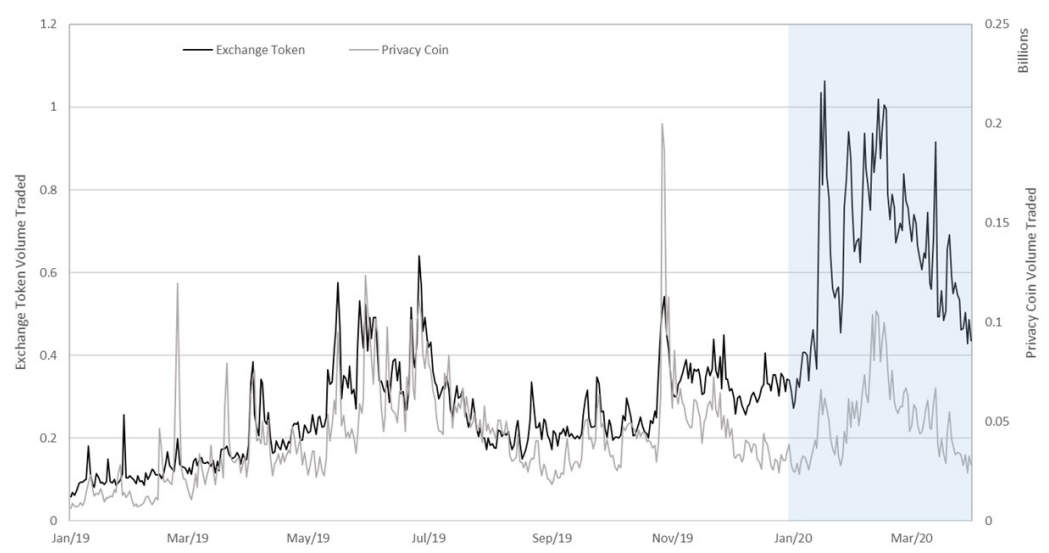

Note: Data is presented for the period 1 January 2019 through 31 March 2020. The shaded. The shaded region represents the WHO-announced onset of the COVID-19 pandemic. 
Figure 3: Social media data relating to cryptocurrency and COVID-19

a) Total number of tweets

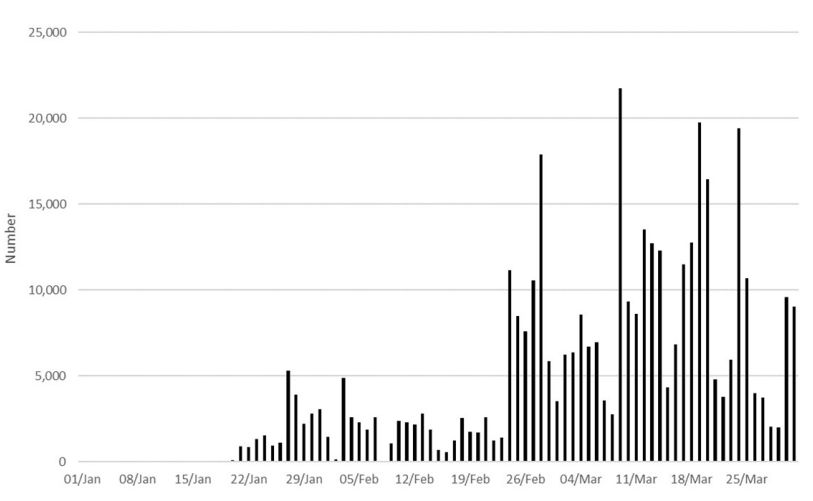

b) Total number of retweets

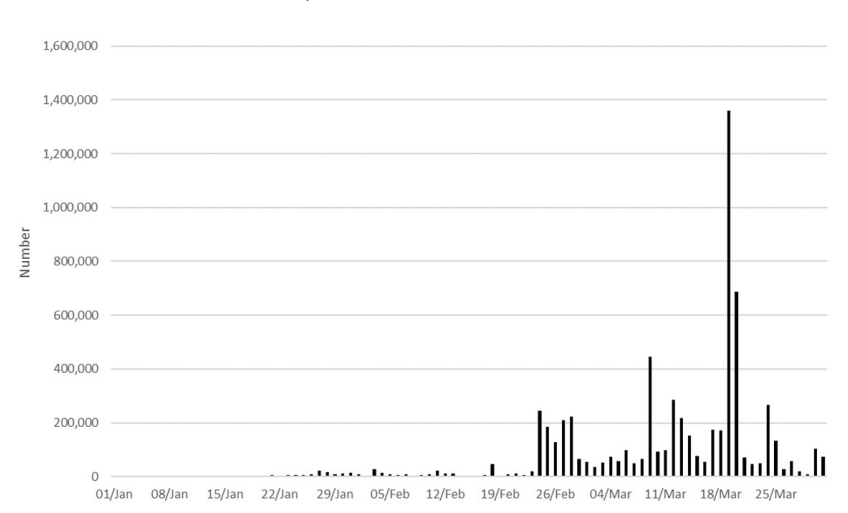

b) Total number of likes

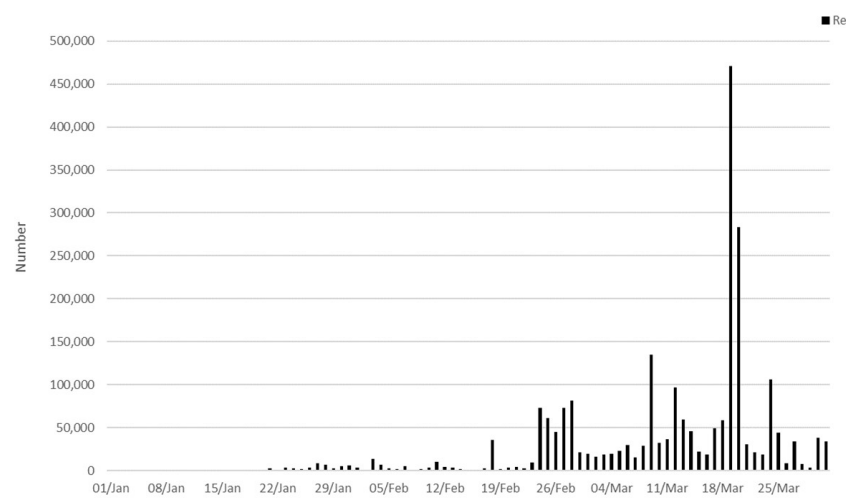

Note: Data is presented for the period 1 January 2020 through 31 March 2020. 
Figure 4: Sentiment relating to cryptocurrency and COVID-19

a) Polarity

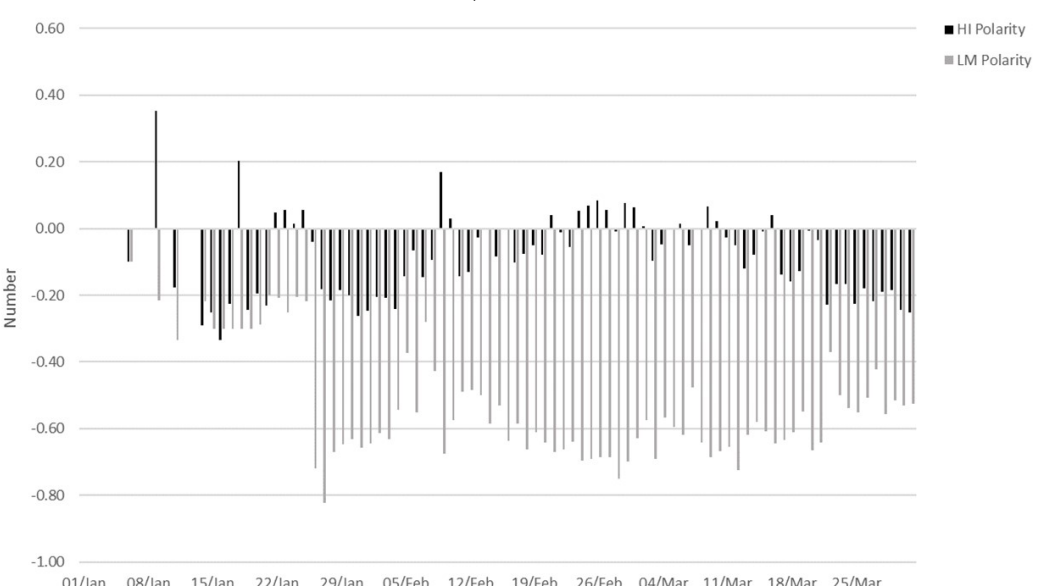

b) Subjectivity

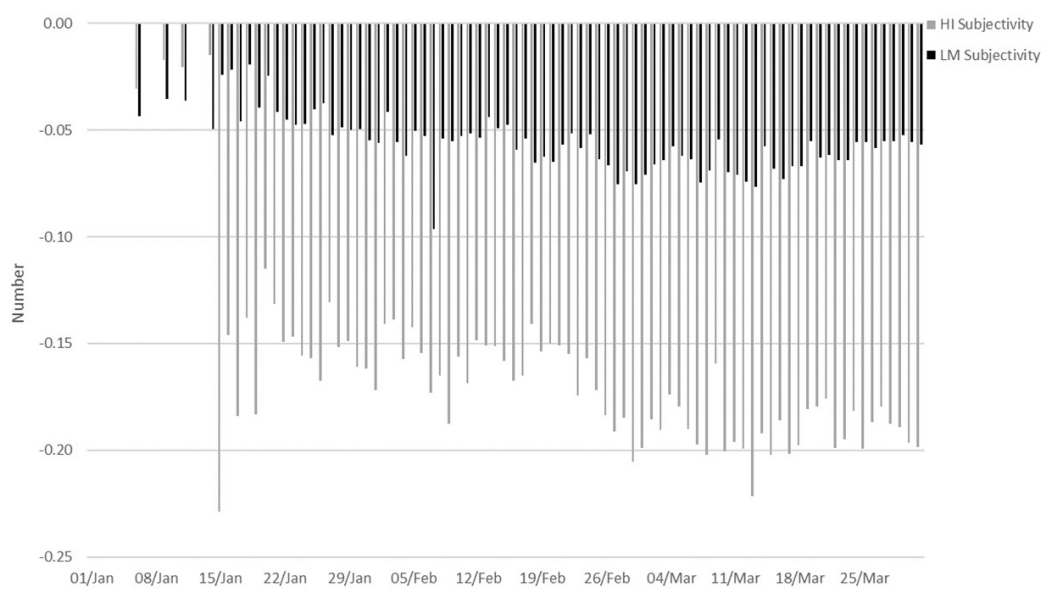

Note: Data is presented for the period 1 January 2020 through 31 March 2020. 
Table 1: Summary statistics based on selected cryptocurrencies

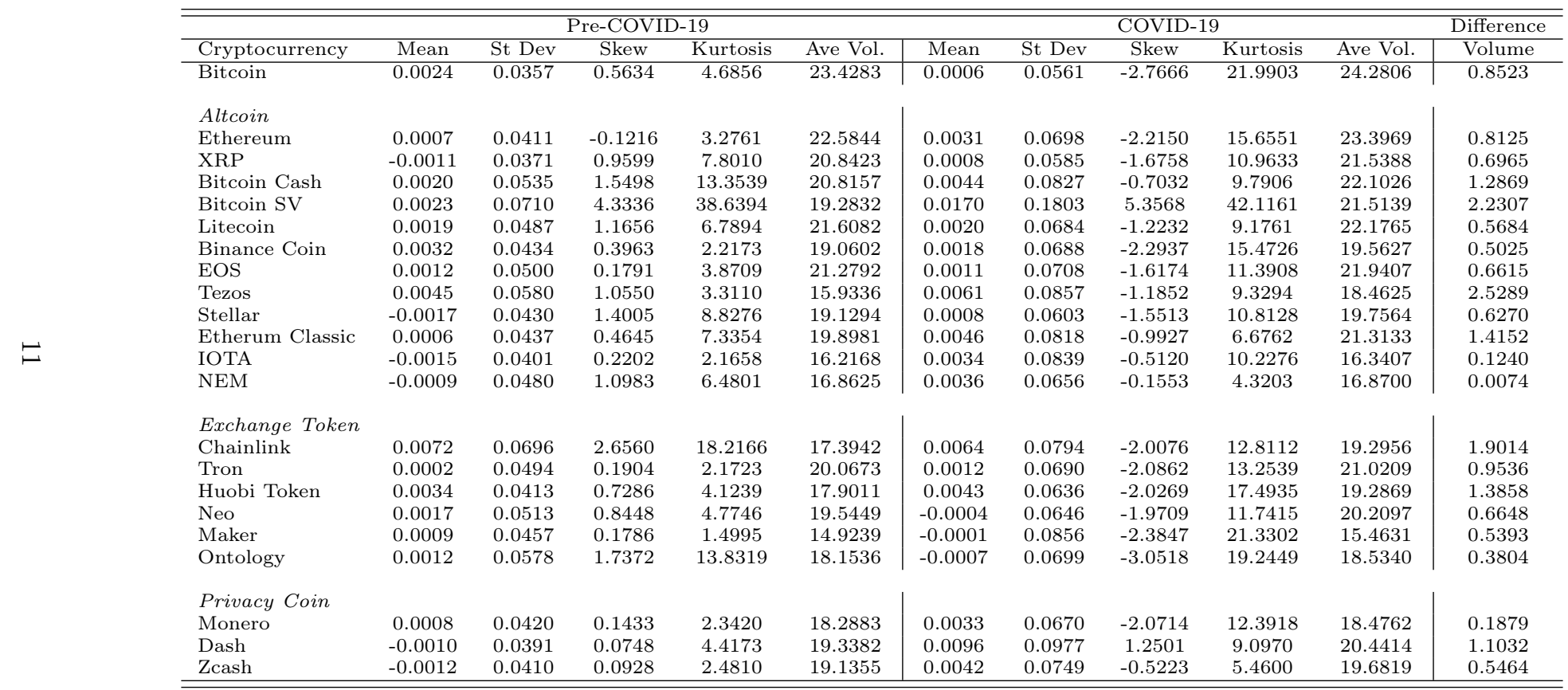

Note: For brevity, some additional results have been omitted from the above table and are available from the authors on request. 
Table 2: GARCH-calculated polarity influence based on COVID-19 pandemic

\begin{tabular}{|c|c|c|c|c|c|c|c|c|}
\hline \multirow[b]{2}{*}{ Cryptocurrency } & \multicolumn{4}{|c|}{ HI Polarity } & \multicolumn{4}{|c|}{ LM Polarity } \\
\hline & Volume & Polarity & $\mathrm{ARCH}$ & GARCH & Volume & Polarity & $\mathrm{ARCH}$ & GARCH \\
\hline Bitcoin & $\begin{array}{l}0.0001^{*} \\
(0.0001)\end{array}$ & $\begin{array}{c}0.0303 \\
(0.0402)\end{array}$ & $\begin{array}{c}0.1670^{* * *} \\
(0.0460)\end{array}$ & $\begin{array}{c}0.7291^{* * *} \\
(0.0615)\end{array}$ & $\begin{array}{l}0.0001^{*} \\
(0.0001)\end{array}$ & $\begin{array}{l}0.0166^{* *} \\
(0.0069)\end{array}$ & $\begin{array}{c}0.2393^{* * *} \\
(0.0547)\end{array}$ & $\begin{array}{c}0.6891^{* * *} \\
(0.0569)\end{array}$ \\
\hline \multicolumn{9}{|l|}{ Altcoin } \\
\hline Ethereum & $\begin{array}{c}0.0001 \\
(0.0001)\end{array}$ & $\begin{array}{c}0.0656 \\
(0.0479)\end{array}$ & $\begin{array}{c}0.1198 * * * \\
(0.0435)\end{array}$ & $\begin{array}{c}0.7475 * * * \\
(0.0853)\end{array}$ & $\begin{array}{c}0.0000 \\
(0.0001)\end{array}$ & $\begin{array}{c}0.0194 * * \\
(0.0084)\end{array}$ & $\begin{array}{c}0.1314^{* * * *} \\
(0.0432)\end{array}$ & $\begin{array}{c}0.7422^{* * * *} \\
(0.0813)\end{array}$ \\
\hline $\mathrm{XRP}$ & $\begin{array}{l}-0.0001 \\
(0.0001)\end{array}$ & $\begin{array}{c}0.0972 * * * \\
(0.0291)\end{array}$ & $\begin{array}{c}0.1654 * * * \\
(0.0410)\end{array}$ & $\begin{array}{c}0.4291 * * * \\
(0.1034)\end{array}$ & $\begin{array}{l}-0.0001 \\
(0.0001)\end{array}$ & $\begin{array}{l}0.0112^{*} \\
(0.0071)\end{array}$ & $\begin{array}{c}0.1725^{* * *} * \\
(0.0470)\end{array}$ & $\begin{array}{c}0.4110^{* * * *} \\
(0.1030)\end{array}$ \\
\hline Bitcoin Cash & $\begin{array}{c}0.0001 \\
(0.0002)\end{array}$ & $\begin{array}{l}0.0770^{*} \\
(0.0572)\end{array}$ & $\begin{array}{c}0.1070 * * * \\
(0.0422)\end{array}$ & $\begin{array}{c}0.5231^{* * *} * \\
(0.1687)\end{array}$ & $\begin{array}{c}0.0001 \\
(0.0002)\end{array}$ & $\begin{array}{c}0.0121 \\
(0.0105)\end{array}$ & $\begin{array}{c}0.1143^{* * *} \\
(0.0444)\end{array}$ & $\begin{array}{c}0.5090^{* * *} * \\
(0.1639)\end{array}$ \\
\hline Bitcoin SV & $\begin{array}{c}0.0000 \\
(0.0001)\end{array}$ & $\begin{array}{c}0.2504^{* * *} * \\
(0.0195)\end{array}$ & $\begin{array}{c}0.5898 * * * \\
(0.0698)\end{array}$ & $\begin{array}{c}0.6395 * * * \\
(0.0223)\end{array}$ & $\begin{array}{c}0.0000 \\
(0.0001)\end{array}$ & $\begin{array}{c}0.0554^{* * *} \\
(0.0069)\end{array}$ & $\begin{array}{c}0.5723 * * * \\
(0.0694)\end{array}$ & $\begin{array}{c}0.6633^{* * *} \\
(0.0226)\end{array}$ \\
\hline Litecoin & $\begin{array}{l}0.0001^{*} \\
(0.0001)\end{array}$ & $\begin{array}{c}0.0512^{* * * *} \\
(0.0194)\end{array}$ & $\begin{array}{c}0.0528 * * * \\
(0.0284)\end{array}$ & $\begin{array}{c}0.6495 * * * \\
(0.1439)\end{array}$ & $\begin{array}{l}0.0001^{*} \\
(0.0001)\end{array}$ & $\begin{array}{c}0.0015 \\
(0.0103)\end{array}$ & $\begin{array}{c}0.0543^{* * *} * \\
(0.0281)\end{array}$ & $\begin{array}{c}0.6520^{* * * *} \\
(0.1386)\end{array}$ \\
\hline EOS & $\begin{array}{c}0.0000 \\
(0.0001)\end{array}$ & $\begin{array}{c}0.0488 \\
(0.0589)\end{array}$ & $\begin{array}{c}0.0551 * * * \\
(0.0104)\end{array}$ & $\begin{array}{c}0.9406^{* * *} * \\
(0.0180)\end{array}$ & $\begin{array}{c}0.0000 \\
(0.0001)\end{array}$ & $\begin{array}{c}0.0042 \\
(0.0088)\end{array}$ & $\begin{array}{c}0.0544^{* * *} * \\
(0.0103)\end{array}$ & $\begin{array}{c}0.9418^{* * * *} \\
(0.0178)\end{array}$ \\
\hline Tezos & $\begin{array}{l}0.0002^{*} \\
(0.0002)\end{array}$ & $\begin{array}{l}0.0620^{*} \\
(0.0421)\end{array}$ & $\begin{array}{c}0.1143^{* * *} * \\
(0.0222)\end{array}$ & $\begin{array}{c}0.8493^{* * *} * \\
(0.0283)\end{array}$ & $\begin{array}{c}0.0001 \\
(0.0002)\end{array}$ & $\begin{array}{c}0.0289^{* *} \\
(0.0105)\end{array}$ & $\begin{array}{c}0.1290^{* * *} * \\
(0.0217)\end{array}$ & $\begin{array}{c}0.8391^{* * *} * \\
(0.0277)\end{array}$ \\
\hline Stellar & $\begin{array}{l}-0.0001 \\
(0.0001)\end{array}$ & $\begin{array}{c}0.0938^{* *} \\
(0.0427)\end{array}$ & $\begin{array}{c}0.2853^{* * * *} \\
(0.0375)\end{array}$ & $\begin{array}{c}0.5886 * * * \\
(0.0393)\end{array}$ & $\begin{array}{c}-0.0002^{* *} \\
(0.0001)\end{array}$ & $\begin{array}{c}0.0270^{* * * *} \\
(0.0079)\end{array}$ & $\begin{array}{c}0.3246^{* * *} \\
(0.0415)\end{array}$ & $\begin{array}{c}0.5614^{* * *} \\
(0.0408)\end{array}$ \\
\hline Cardano & $\begin{array}{c}0.0001 \\
(0.0001)\end{array}$ & $\begin{array}{l}0.0512^{*} \\
(0.0336)\end{array}$ & $\begin{array}{c}0.2613^{* * *} \\
(0.0627)\end{array}$ & $\begin{array}{c}0.4191 * * * \\
(0.0930)\end{array}$ & $\begin{array}{c}0.0000 \\
(0.0002)\end{array}$ & $\begin{array}{c}0.0271 * * * \\
(0.0078)\end{array}$ & $\begin{array}{c}0.3778^{* * *} \\
(0.0682)\end{array}$ & $\begin{array}{c}0.3618^{* * * *} \\
(0.0844)\end{array}$ \\
\hline Ethereum Classic & $\begin{array}{c}0.0000 \\
(0.0001)\end{array}$ & $\begin{array}{c}0.0333 \\
(0.0443)\end{array}$ & $\begin{array}{c}0.1631 * * * \\
(0.0631)\end{array}$ & $\begin{array}{c}0.5805^{* * *} \\
(0.1232)\end{array}$ & $\begin{array}{l}0.0000 \\
(0.0002)\end{array}$ & $\begin{array}{l}0.0117^{*} \\
(0.0097)\end{array}$ & $\begin{array}{c}0.1714^{* * *} \\
(0.0675)\end{array}$ & $\begin{array}{c}0.5743^{* * *} \\
(0.1200)\end{array}$ \\
\hline Iota & $\begin{array}{c}-0.0001 \\
(0.0001)\end{array}$ & $\begin{array}{c}0.0487 \\
(0.0712)\end{array}$ & $\begin{array}{c}0.1715^{* * *} \\
(0.0436)\end{array}$ & $\begin{array}{c}0.7640^{* *} \\
(0.0609)\end{array}$ & $\begin{array}{c}-0.0001^{*} \\
(0.0001)\end{array}$ & $\begin{array}{c}0.0390 * * * \\
(0.0086)\end{array}$ & $\begin{array}{c}0.1283^{* * *} \\
(0.0285)\end{array}$ & $\begin{array}{c}0.8463^{* * *} * \\
(0.0367)\end{array}$ \\
\hline NEM & $\begin{array}{c}-0.0002^{* *} \\
(0.0001)\end{array}$ & $\begin{array}{l}0.0435 * \\
(0.0355)\end{array}$ & $\begin{array}{c}0.3434^{* * *} \\
(0.0672)\end{array}$ & $\begin{array}{c}0.4072^{* * *} \\
(0.0826)\end{array}$ & $\begin{array}{c}-0.0002^{* *} \\
(0.0001)\end{array}$ & $\begin{array}{l}0.0132^{*} \\
(0.0075)\end{array}$ & $\begin{array}{c}0.3261 * * * \\
(0.0637)\end{array}$ & $\begin{array}{c}0.4047^{* * *} \\
(0.0835)\end{array}$ \\
\hline Exchange Token & & & & & & & & \\
\hline Chainlink & $\begin{array}{c}0.0002 \\
(0.0002)\end{array}$ & $\begin{array}{l}0.0821^{*} \\
(0.0536)\end{array}$ & $\begin{array}{c}0.1006 * * * \\
(0.0287)\end{array}$ & $\begin{array}{c}0.9037^{* *} \\
(0.0253)\end{array}$ & $\begin{array}{c}0.0002 \\
(0.0002)\end{array}$ & $\begin{array}{c}0.0236 * * * \\
(0.0087)\end{array}$ & $\begin{array}{c}0.2093 * * * \\
(0.0352)\end{array}$ & $\begin{array}{c}0.8139 * * * \\
(0.0323)\end{array}$ \\
\hline Tron & $\begin{array}{c}0.0000 \\
(0.0001)\end{array}$ & $\begin{array}{c}0.0611 \\
(0.0561)\end{array}$ & $\begin{array}{c}0.1714^{* * * *} \\
(0.0425)\end{array}$ & $\begin{array}{c}0.7460^{* * * *} \\
(0.0588)\end{array}$ & $\begin{array}{l}-0.0001 \\
(0.0001)\end{array}$ & $\begin{array}{c}0.0293^{* * *} * \\
(0.0085)\end{array}$ & $\begin{array}{c}0.1942^{* * *} \\
(0.0418)\end{array}$ & $\begin{array}{c}0.7478^{* * *} \\
(0.0505)\end{array}$ \\
\hline Huobitoken & $\begin{array}{l}0.0001 \\
(0.0001)\end{array}$ & $\begin{array}{c}0.0187 \\
(0.0518)\end{array}$ & $\begin{array}{c}0.0782^{* * * *} \\
(0.0156)\end{array}$ & $\begin{array}{c}0.8994 * * * \\
(0.0172)\end{array}$ & $\begin{array}{c}0.0002^{* *} \\
(0.0001)\end{array}$ & $\begin{array}{c}0.0200 * * * \\
(0.0035)\end{array}$ & $\begin{array}{c}0.6078^{* *} \\
(0.1812)\end{array}$ & $\begin{array}{c}0.0934 * * * \\
(0.0282)\end{array}$ \\
\hline Neo & $\begin{array}{c}0.0001 \\
(0.0001)\end{array}$ & $\begin{array}{c}0.0572 \\
(0.0456)\end{array}$ & $\begin{array}{c}0.2584^{* * *} * \\
(0.0585)\end{array}$ & $\begin{array}{c}0.5755 * * * \\
(0.0727)\end{array}$ & $\begin{array}{c}0.0000 \\
(0.0001)\end{array}$ & $\begin{array}{c}0.0234^{* * *} * \\
(0.0085)\end{array}$ & $\begin{array}{c}0.3162^{* * *} \\
(0.0636)\end{array}$ & $\begin{array}{c}0.5290^{* * *} \\
(0.0775)\end{array}$ \\
\hline Maker & $\begin{array}{c}0.0001 \\
(0.0002)\end{array}$ & $\begin{array}{c}0.0415 \\
(0.0664)\end{array}$ & $\begin{array}{c}0.1573^{* * *} \\
(0.0453)\end{array}$ & $\begin{array}{c}0.7957^{* * *} \\
(0.0643)\end{array}$ & $\begin{array}{c}0.0001 \\
(0.0002)\end{array}$ & $\begin{array}{l}0.0106^{*} \\
(0.0096)\end{array}$ & $\begin{array}{c}0.1544^{* * *} \\
(0.0426)\end{array}$ & $\begin{array}{c}0.7992^{* * *} * \\
(0.0627)\end{array}$ \\
\hline Ontology & $\begin{array}{c}0.0000 \\
(0.0002)\end{array}$ & $\begin{array}{c}0.0292 \\
(0.0530)\end{array}$ & $\begin{array}{c}0.3099 * * * \\
(0.0434)\end{array}$ & $\begin{array}{c}0.5784^{* * *} * \\
(0.0595)\end{array}$ & $\begin{array}{c}0.0000 \\
(0.0002)\end{array}$ & $\begin{array}{c}0.0227^{*} \\
(0.0118)\end{array}$ & $\begin{array}{c}0.3435 * * * \\
(0.0457)\end{array}$ & $\begin{array}{c}0.5752^{* * *} * \\
(0.0506)\end{array}$ \\
\hline Privacy Coin & & & & & & & & \\
\hline Monero & $\begin{array}{c}0.0001 \\
(0.0001)\end{array}$ & $\begin{array}{l}0.0505^{*} \\
(0.0302)\end{array}$ & $\begin{array}{c}0.1826 * * * \\
(0.0508)\end{array}$ & $\begin{array}{c}0.7273 * * * \\
(0.0663)\end{array}$ & $\begin{array}{l}0.0001^{*} \\
(0.0001)\end{array}$ & $\begin{array}{c}0.0209 * * * \\
(0.0089)\end{array}$ & $\begin{array}{c}0.2078^{* * *} * \\
(0.0520)\end{array}$ & $\begin{array}{c}0.7142^{* * *} \\
(0.0642)\end{array}$ \\
\hline Dash & $\begin{array}{c}0.0000 \\
(0.0001)\end{array}$ & $\begin{array}{l}0.0716^{*} \\
(0.0472)\end{array}$ & $\begin{array}{c}0.4432 * * * \\
(0.0710)\end{array}$ & $\begin{array}{c}0.5604^{* *} \\
(0.0455)\end{array}$ & $\begin{array}{c}0.0000 \\
(0.0001)\end{array}$ & $\begin{array}{c}0.0223^{* * *} * \\
(0.0069)\end{array}$ & $\begin{array}{c}0.6084^{* * * *} \\
(0.0972)\end{array}$ & $\begin{array}{c}0.4376^{* * * *} \\
(0.0585)\end{array}$ \\
\hline Zcash & $\begin{array}{c}0.0000 \\
(0.0001)\end{array}$ & $\begin{array}{l}0.0676^{*} \\
(0.0490)\end{array}$ & $\begin{array}{c}0.2362^{* * *} \\
(0.0470)\end{array}$ & $\begin{array}{c}0.6694 * * * \\
(0.0554)\end{array}$ & $\begin{array}{c}0.0000 \\
(0.0001)\end{array}$ & $\begin{array}{l}0.0243^{*} \\
(0.0105)\end{array}$ & $\begin{array}{c}0.2815^{* * *} \\
(0.0597)\end{array}$ & $\begin{array}{c}0.6171 * * * \\
(0.0692)\end{array}$ \\
\hline
\end{tabular}

Note: $* * *, * *$ and $*$ denote significance at the $1 \%, 5 \%$ and $10 \%$ levels. For brevity, some additional results have been omitted from the above table and are available from the authors on request. 
Table 3: GARCH-calculated subjectivity influence based on COVID-19 pandemic

\begin{tabular}{|c|c|c|c|c|c|c|c|c|}
\hline \multirow[b]{2}{*}{ Cryptocurrency } & \multicolumn{4}{|c|}{ HI Subjectivity } & \multicolumn{4}{|c|}{ LM Subjectivity } \\
\hline & Volume & Subjectivity & $\mathrm{ARCH}$ & GARCH & Volume & Subjectivity & $\mathrm{ARCH}$ & GARCH \\
\hline Bitcoin & $\begin{array}{l}0.0001^{*} \\
(0.0001)\end{array}$ & $\begin{array}{c}0.0870^{* * *} \\
(0.0241)\end{array}$ & $\begin{array}{c}0.2878^{* * *} \\
(0.0601)\end{array}$ & $\begin{array}{c}0.6562^{* * *} \\
(0.0573)\end{array}$ & $\begin{array}{c}0.0000 \\
(0.0001)\end{array}$ & $\begin{array}{c}0.2987^{* * *} \\
(0.0700)\end{array}$ & $\begin{array}{c}0.3066^{* * *} \\
(0.0619)\end{array}$ & $\begin{array}{c}0.6416^{* * *} \\
(0.0573)\end{array}$ \\
\hline \multicolumn{9}{|l|}{ Altcoin } \\
\hline Ethereum & $\begin{array}{c}0.0000 \\
(0.0001)\end{array}$ & $\begin{array}{c}0.3214^{* * *} \\
(0.1021)\end{array}$ & $\begin{array}{c}0.1489 * * * \\
(0.0459)\end{array}$ & $\begin{array}{c}0.7266^{* * *} \\
(0.0818)\end{array}$ & $\begin{array}{c}0.0000 \\
(0.0001)\end{array}$ & $\begin{array}{c}0.3214^{* * *} \\
(0.1021)\end{array}$ & $\begin{array}{c}0.1489 * * * \\
(0.0459)\end{array}$ & $\begin{array}{c}0.7266^{* * *} * \\
(0.0818)\end{array}$ \\
\hline $\mathrm{XRP}$ & $\begin{array}{c}0.0002 * * \\
(0.0001)\end{array}$ & $\begin{array}{c}0.1718^{* * *} * \\
(0.0180)\end{array}$ & $\begin{array}{c}0.5783 * * * \\
(0.0868)\end{array}$ & $\begin{array}{c}0.3111^{* * *} * \\
(0.0736)\end{array}$ & $\begin{array}{l}-0.0001 \\
(0.0001)\end{array}$ & $\begin{array}{c}0.0733 \\
(0.0939)\end{array}$ & $\begin{array}{c}0.1642^{* * *} \\
(0.0450)\end{array}$ & $\begin{array}{c}0.4151^{* *} \\
(0.1039)\end{array}$ \\
\hline Bitcoin Cash & $\begin{array}{c}0.0001 \\
(0.0002)\end{array}$ & $\begin{array}{c}0.0691^{* * *} \\
(0.0052)\end{array}$ & $\begin{array}{c}0.0935 * * * \\
(0.02011)\end{array}$ & $\begin{array}{c}0.3471^{* * *} * \\
(0.0938)\end{array}$ & $\begin{array}{c}0.0001 \\
(0.0002)\end{array}$ & $\begin{array}{c}0.0753^{* * *} * \\
(0.0139)\end{array}$ & $\begin{array}{c}0.1117 * * * \\
(0.0432)\end{array}$ & $\begin{array}{c}0.5113^{* *} \\
(0.1639)\end{array}$ \\
\hline Bitcoin SV & $\begin{array}{l}0.0001^{* *} \\
(0.0001)\end{array}$ & $\begin{array}{c}0.4171 * * * \\
(0.0590)\end{array}$ & $\begin{array}{c}0.4156 * * * \\
(0.0782)\end{array}$ & $\begin{array}{c}0.5927 * * * \\
(0.0578)\end{array}$ & $\begin{array}{c}0.0000 \\
(0.0001)\end{array}$ & $\begin{array}{c}0.6576 * * * \\
(0.0975)\end{array}$ & $\begin{array}{c}0.5798^{* * *} \\
(0.0698)\end{array}$ & $\begin{array}{c}0.6638^{* * * *} \\
(0.0231)\end{array}$ \\
\hline Litecoin & $\begin{array}{l}0.0001^{*} \\
(0.0001)\end{array}$ & $\begin{array}{c}0.0008 \\
(0.0400)\end{array}$ & $\begin{array}{l}0.0538^{*} \\
(0.0278)\end{array}$ & $\begin{array}{c}0.6515 * * * \\
(0.1391)\end{array}$ & $\begin{array}{l}0.0001^{*} \\
(0.0001)\end{array}$ & $\begin{array}{c}0.0277 \\
(0.1198)\end{array}$ & $\begin{array}{l}0.0528^{*} \\
(0.0271)\end{array}$ & $\begin{array}{c}0.6493 * * * \\
(0.1391)\end{array}$ \\
\hline EOS & $\begin{array}{c}0.0000 \\
(0.0001)\end{array}$ & $\begin{array}{c}0.0118 \\
(0.0364)\end{array}$ & $\begin{array}{c}0.0541 * * * \\
(0.0102)\end{array}$ & $\begin{array}{c}0.9426 * * * \\
(0.0178)\end{array}$ & $\begin{array}{c}0.0000 \\
(0.0001)\end{array}$ & $\begin{array}{c}0.0093 \\
(0.1096)\end{array}$ & $\begin{array}{c}0.0533 * * * \\
(0.0102)\end{array}$ & $\begin{array}{c}0.9445^{* * *} * \\
(0.0177)\end{array}$ \\
\hline Tezos & $\begin{array}{c}0.0001 \\
(0.0002)\end{array}$ & $\begin{array}{c}0.1283^{* *} \\
(0.0419)\end{array}$ & $\begin{array}{c}0.1326^{* * *} \\
(0.0221)\end{array}$ & $\begin{array}{c}0.8350^{* * *} \\
(0.0283)\end{array}$ & $\begin{array}{c}0.0001 \\
(0.0002)\end{array}$ & $\begin{array}{c}0.4328^{* * *} \\
(0.1319)\end{array}$ & $\begin{array}{c}0.1349 * * * \\
(0.0226)\end{array}$ & $\begin{array}{c}0.8316^{* * *} \\
(0.0289)\end{array}$ \\
\hline Stellar & $\begin{array}{c}-0.0002^{* *} \\
(0.0001)\end{array}$ & $\begin{array}{c}0.1053^{* * *} \\
(0.0294)\end{array}$ & $\begin{array}{c}0.3280 * * * \\
(0.0418)\end{array}$ & $\begin{array}{c}0.5568 * * * \\
(0.0431)\end{array}$ & $\begin{array}{c}0.0002 * * * \\
(0.0001)\end{array}$ & $\begin{array}{c}0.2954 * * * \\
(0.0961)\end{array}$ & $\begin{array}{c}0.3272^{* * *} \\
(0.0418)\end{array}$ & $\begin{array}{c}0.5565^{* * *} \\
(0.0447)\end{array}$ \\
\hline Cardano & $\begin{array}{c}0.0000 \\
(0.0002)\end{array}$ & $\begin{array}{c}0.0984^{* * *} * \\
(0.0305)\end{array}$ & $\begin{array}{c}0.3893^{* * *} \\
(0.0699)\end{array}$ & $\begin{array}{c}0.3533^{* * *} \\
(0.0843)\end{array}$ & $\begin{array}{c}0.0000 \\
(0.0002)\end{array}$ & $\begin{array}{c}0.2885 * * * \\
(0.0922)\end{array}$ & $\begin{array}{c}0.3874^{* * *} \\
(0.0703)\end{array}$ & $\begin{array}{c}0.3502^{* * *} \\
(0.0837)\end{array}$ \\
\hline Ethereum Classic & $\begin{array}{c}0.0001 \\
(0.0002)\end{array}$ & $\begin{array}{c}0.0674^{* *} \\
(0.0389)\end{array}$ & $\begin{array}{c}0.1756 * * * \\
(0.0689)\end{array}$ & $\begin{array}{c}0.5808^{* * *} \\
(0.1200)\end{array}$ & $\begin{array}{c}0.0001 \\
(0.0002)\end{array}$ & $\begin{array}{c}0.2575^{*} \\
(0.1120)\end{array}$ & $\begin{array}{c}0.1675^{* * *} * \\
(0.0655)\end{array}$ & $\begin{array}{c}0.5919^{* * * *} \\
(0.1172)\end{array}$ \\
\hline Iota & $\begin{array}{l}-0.0001^{*} \\
(0.0001)\end{array}$ & $\begin{array}{c}0.1621^{* * *} \\
(0.0361)\end{array}$ & $\begin{array}{c}0.1430 * * * \\
(0.0320)\end{array}$ & $\begin{array}{c}0.8271 * * * \\
(0.0411)\end{array}$ & $\begin{array}{c}-0.0001^{*} \\
(0.0001)\end{array}$ & $\begin{array}{c}0.4512^{* * *} \\
(0.1122)\end{array}$ & $\begin{array}{c}0.1405^{* * *} * \\
(0.0318)\end{array}$ & $\begin{array}{c}0.8308^{* * *} \\
(0.0410)\end{array}$ \\
\hline NEM & $\begin{array}{c}-0.0002^{*} \\
(0.0001)\end{array}$ & $\begin{array}{l}0.0447^{*} \\
(0.0273)\end{array}$ & $\begin{array}{c}0.3202^{* * *} \\
(0.0625)\end{array}$ & $\begin{array}{c}0.4084^{* * *} \\
(0.0840)\end{array}$ & $\begin{array}{c}-0.0002^{* * *} \\
(0.0001)\end{array}$ & $\begin{array}{c}0.0917 \\
(0.0795)\end{array}$ & $\begin{array}{c}0.3214^{* * *} \\
(0.0635)\end{array}$ & $\begin{array}{c}0.4073^{* * *} * \\
(0.0838)\end{array}$ \\
\hline Exchange Token & & & & & & & & \\
\hline Chainlink & $\begin{array}{l}0.0002^{*} \\
(0.0002)\end{array}$ & $\begin{array}{l}0.0942^{*} \\
(0.0520)\end{array}$ & $\begin{array}{c}0.1936^{* * *} \\
(0.0357)\end{array}$ & $\begin{array}{c}0.8201 * * * \\
(0.0315)\end{array}$ & $\begin{array}{c}0.0000 \\
(0.0002)\end{array}$ & $\begin{array}{c}0.4549 * * * \\
(0.0973)\end{array}$ & $\begin{array}{c}0.2368 * * * \\
(0.0413)\end{array}$ & $\begin{array}{c}0.7972 * * * \\
(0.0341)\end{array}$ \\
\hline Tron & $\begin{array}{c}-0.0001^{*} \\
(0.0001)\end{array}$ & $\begin{array}{c}0.1279 * * * \\
(0.0312)\end{array}$ & $\begin{array}{c}0.2055^{* * *} * \\
(0.0420)\end{array}$ & $\begin{array}{c}0.7408^{* * *} * \\
(0.0491)\end{array}$ & $\begin{array}{c}-0.0001^{*} \\
(0.0001)\end{array}$ & $\begin{array}{c}0.3920^{* * *} * \\
(0.0964)\end{array}$ & $\begin{array}{c}0.2026^{* * *} * \\
(0.0415)\end{array}$ & $\begin{array}{c}0.7426^{* * *} * \\
(0.0497)\end{array}$ \\
\hline Huobitoken & $\begin{array}{l}0.0002^{* *} \\
(0.0001)\end{array}$ & $\begin{array}{c}0.0760 * * * \\
(0.0114)\end{array}$ & $\begin{array}{c}0.2815^{* * *} \\
(0.1807)\end{array}$ & $\begin{array}{c}0.0716^{* *} \\
(0.0249)\end{array}$ & $\begin{array}{c}0.0002^{* *} \\
(0.0001)\end{array}$ & $\begin{array}{c}0.2462 * * * \\
(0.0323)\end{array}$ & $\begin{array}{c}0.7338^{* * *} * \\
(0.1754)\end{array}$ & $\begin{array}{l}0.0456^{*} \\
(0.0209)\end{array}$ \\
\hline $\mathrm{Neo}$ & $\begin{array}{l}0.0000 \\
(0.0001)\end{array}$ & $\begin{array}{c}0.1032 * * * \\
(0.0303)\end{array}$ & $\begin{array}{c}0.3478 * * * \\
(0.0685)\end{array}$ & $\begin{array}{c}0.4998^{* * *} \\
(0.0805)\end{array}$ & $\begin{array}{l}0.0000 \\
(0.0001)\end{array}$ & $\begin{array}{c}0.2876 * * * \\
(0.0914)\end{array}$ & $\begin{array}{c}0.3325^{* * *} \\
(0.0665)\end{array}$ & $\begin{array}{c}0.5132^{* * *} \\
(0.0796)\end{array}$ \\
\hline Maker & $\begin{array}{c}0.0001 \\
(0.0002)\end{array}$ & $\begin{array}{c}0.0511 \\
(0.0370)\end{array}$ & $\begin{array}{l}0.1558^{* *} \\
(0.0427)\end{array}$ & $\begin{array}{c}0.7979 * * * \\
(0.0630)\end{array}$ & $\begin{array}{c}0.0000 \\
(0.0002)\end{array}$ & $\begin{array}{c}0.1759 \\
(0.1138)\end{array}$ & $\begin{array}{c}0.1565 * * * \\
(0.0427)\end{array}$ & $\begin{array}{c}0.7978^{* * *} * \\
(0.0627)\end{array}$ \\
\hline Ontology & $\begin{array}{c}0.0000 \\
(0.0002)\end{array}$ & $\begin{array}{c}0.1018^{* * *} \\
(0.0422)\end{array}$ & $\begin{array}{c}0.3618^{* * *} \\
(0.0473)\end{array}$ & $\begin{array}{c}0.5671^{* * *} \\
(0.0515)\end{array}$ & $\begin{array}{c}0.0000 \\
(0.0002)\end{array}$ & $\begin{array}{c}0.3591^{* * *} \\
(0.1188)\end{array}$ & $\begin{array}{c}0.3823 * * * \\
(0.0496)\end{array}$ & $\begin{array}{c}0.5548^{* * *} \\
(0.0533)\end{array}$ \\
\hline Privacy Coin & & & & & & & & \\
\hline Monero & $\begin{array}{l}0.0001^{*} \\
(0.0001)\end{array}$ & $\begin{array}{c}0.0972 * * * \\
(0.0328)\end{array}$ & $\begin{array}{c}0.2170 * * * \\
(0.0527)\end{array}$ & $\begin{array}{c}0.7060^{* * *} \\
(0.0642)\end{array}$ & $\begin{array}{l}0.0001^{*} \\
(0.0001)\end{array}$ & $\begin{array}{c}0.2699^{* *} \\
(0.1011)\end{array}$ & $\begin{array}{c}0.2178^{* * *} \\
(0.0531)\end{array}$ & $\begin{array}{c}0.7052^{* * *} \\
(0.0644)\end{array}$ \\
\hline Dash & $\begin{array}{c}0.0000 \\
(0.0001)\end{array}$ & $\begin{array}{c}0.0791 * * * \\
(0.0228)\end{array}$ & $\begin{array}{c}0.5972 * * * \\
(0.0953)\end{array}$ & $\begin{array}{c}0.4436^{* * *} \\
(0.0576)\end{array}$ & $\begin{array}{c}0.0000 \\
(0.0001)\end{array}$ & $\begin{array}{c}0.2233 * * * \\
(0.0662)\end{array}$ & $\begin{array}{c}0.6198 * * * \\
(0.0991)\end{array}$ & $\begin{array}{c}0.4281^{* * *} * \\
(0.0587)\end{array}$ \\
\hline Zcash & $\begin{array}{c}0.0000 \\
(0.0001)\end{array}$ & $\begin{array}{l}0.0831^{*} \\
(0.0352)\end{array}$ & $\begin{array}{c}0.2855^{* * *} \\
(0.0590)\end{array}$ & $\begin{array}{c}0.6134^{* * *} * \\
(0.0671)\end{array}$ & $\begin{array}{c}0.0000 \\
(0.0001)\end{array}$ & $\begin{array}{l}0.1801^{*} \\
(0.1029)\end{array}$ & $\begin{array}{c}0.2764^{* * *} \\
(0.0549)\end{array}$ & $\begin{array}{c}0.6262^{* * *} \\
(0.0650)\end{array}$ \\
\hline
\end{tabular}

Note: $* * *, * *$ and $*$ denote significance at the $1 \%, 5 \%$ and $10 \%$ levels. For brevity, some additonal results have been omitted from the above table and are available from the authors on request. 